\title{
KIF15 is An Oncogene of Prostate Cancer and is A Key Prognostic Factor in Patients with Prostate Cancer
}

Lin Li ( $\square$ lilinurology@163.com )

Tangshan Gongren Hospital

Jing Hao

North China University of Science and Technology

Jin-Xiu Liu

Tangshan Gongren Hospital

Peng-Fei Wang

Tangshan Gongren Hospital

Chao-Fei Zhao

Tangshan Gongren Hospital

Sheng-Yong Cai

Tangshan Gongren Hospital

\section{Research Article}

Keywords: Prostate cancer, KIF15, disease-free survival, therapeutic target.

Posted Date: June 22nd, 2021

DOI: https://doi.org/10.21203/rs.3.rs-575206/v1

License: (9) (i) This work is licensed under a Creative Commons Attribution 4.0 International License. Read Full License 


\section{Abstract \\ Background}

KIF15, a member of kinesin superfamily proteins, has been found that play a of vital role in the carcinogenesis of various malignant tumor. But whether KIF15 can facilitate the evolution of prostate cancer (PCa) is still unknown. This study aims to explore its biological function in PCa cells and its relevance to prognosis and clinical features in PCa patients.

\section{Material and Methods}

KIF15 expression at mRNA and protein level in tumor and normal tissues was detected by quantitative real-time PCR (RT-PCR) and immunohistochemistry. Then the correlations between KIF15 expression and PCa patients' clinical characteristics was analyzed. After inhibiting the expression of KIF15 by shRNA, the role of KIF15 on proliferative capacity of PCa was evaluated by using MTT assay. The function of KIF15 on metastatic potential of PCa was determined by using transwell assay. The prognostic value of KIF15 was determined by using bioinformatics analysis.

\section{Results}

Compared with normal tissues, KIF15 was overexpressed in PCa tissues. After knocking down KIF15 in C4-2 and Lncap cell lines, the proliferation $(P<0.001)$ and invasion $(P<0.001)$ capabilities of tumor cells are significantly reduced compared to the shCON group. The proliferation marker Ki67 and the metastasis-related marker MMP9 were also significantly reduced in two cell lines after silencing KIF15. Except that, increased KIF15 in tumor tissue is associated with clinical stage $(P=0.004)$, seminal vesicle invasion $(P=0.02)$, lymph node metastasis $(P=0.03)$, and poor disease-free survival $(P<0.05)$ in $P C a$ patients.

\section{Conclusions}

The results proved that KIF15 might served as a prognostic factor and therapeutic target in prostate cancer, and play as a vital regulatory factor in tumorigenesis and cancer development of prostate cancer.

\section{Introduction}

Prostate cancer is still a common malignant tumor in male in developed countries, and its morbidity and mortality have gradually increased in recent years[1-3]. In addition, the increasing economic burden and care costs for treating prostate cancer each year have become global issues[4]. Due to the increasing popularity of PSA screening in China, the morbidity of PCa in Chinese male is also increasing, which form a problem that cannot be ignored[5]. Although prostate cancer patients can benefit from radical 
prostatectomy and androgen deprivation treatment, and the overall survival (OS) of PCa patients has continued to increase in recent years, PCa patients inevitably have recurrence and metastasis 3-5 years after receiving the initial treatment. Eventually it progressed to castration-resistant prostate cancer[6]. So far, the molecular mechanism of the occurrence and development of PCa hasn't been fully understood[7, 8]. At the same time, prostate cancer is a heterogeneous disease[9]. With the development of precision medicine, it is urgent to find an oncogenic factor that promotes the pathological development of prostate cancer to provide better treatment strategies for urologists[10].

Kinesin superfamily proteins was known as "hub" protein in microtubule depolymerizing, chromosome segregation and organelle transport[11]. So these proteins play an essential role in cellular morphology, mitosis and meiosis[12,13]. In recent years, more and more studies have pointed out that these proteins are closely related to the tumorigenesis and occurrence of cancer $[14,15]$. Xiong et al. demonstrated that the upregulation of KIF20A promoted cell proliferation and drug resistance via JAK/STAT3 pathway in colorectal cancer[16]. KIF15, which belongs to the Kinesin-12 family, is thought to be related to the proliferation, invasion, metastasis and poor prognosis of various tumor cells[17]. For instance, Wang et al found that suppressing KIF15 expression inhibit proliferation ability of pancreatic cancer effectively by activated MEK-ERK pathway and KIF15 promotes the transformation of tumor cells from G1 phase to S phase by regulating cell cycle related proteins[18]. Except that, Millic et al highlighted that KIF15 inhibitor combined with Eg5 inhibitor is an effective strategy to solve the problem of chemotherapy resistance[19]. But there is few researches with regard to the characterization of KIF15 in the tumorigenesis of prostate cancer.

Therefore, in the present research, we compared the KIF15 expression in PCa tissues and prostatic tissues using immunohistochemical methods. Furthermore, the correlation between the KIF15 expression and the PCa patients prognosis was analyzed by means of bioinformatics. Finally, we used MTT, Transwell, and western blot assay to invest the function of KIF15 in the proliferation and invasion abilities of PCa in vitro. This study aims to prove that KIF15 can be a original therapeutic target for PCa patients and a predictor for prognostic analysis of PCa patients.

\section{Materials And Methods}

\section{Patients information and tissues specimen}

We collected clinical information and tissue samples of patients undergoing radical prostatectomy in our institution from 2016 to 2019. Clinical information includes age, PSA level at initial diagnosis, clinical stage, Gleason score, and whether the seminal vesicles and lymph nodes are invaded. None of these patients received androgen deprivation therapy, chemotherapy or radiation before surgery. All patients signed informed consent. This study was approved by the Ethics Committee of Tangshan Gongren Hospital. The clinical trial registry number is GRYY-LL-2019-40.

\section{Cell lines}


The human PCa cell lines PC-3, C4-2, and Lncap was used in this study, which was purchased from the ATCC (American Type Culture Collection, USA). The human prostatic hyperplasia cell line BPH-1 was purchased from the HonSun company (Shanghai). The PC-3, C4-2, Lncap and BPH-1 cell line was cultured in RPMI 1640 (Biological Industries, 01-100-1A, Israel) medium containing 10\% fetal bovine serum (Biological Industries, 04-400-1A, Israel) in a $37^{\circ} \mathrm{C}$ incubator containing $5 \%$ carbon dioxide.

\section{Knockdown KIF20A by small hairpin RNAs}

The lentiviral vector which contained short hairpin RNA(shRNA) targeting KIF15 was purchased from Genechem Technology (Shanghai, China). The most efficient shRNA sequence to knock down KIF15 was: 5'-TGAAGTGAAGAGGCTCAAA-3' and the control shRNA sequence was: 5'-TTCTCCGAACGTG TCACGT-3'. According to the instructions, a mixture of $100 \mathrm{nM}$ shRNA and lipofectamine 3000 Transfection Reagent (Invitrogen,L3000015,USA) was added to the serum-free RPMI 1640 medium. Detection of KIF15 knockdown efficiency by Western blot after 48 hours

\section{Quantitative real-time PCR}

Total RNA was extracted from tumor tissues and normal tissues by using Trizol reagent (Invitrogen,15596018,USA).According to the manufacturer's protocol, Reverse Transcription system, which included DEPC water, primer-mix, dNTP-mix, DTT and 5xPrimeScript buffer, was used to reversetranscribed total RNA to generate cDNA. SYBR Premix Ex Taq was performed to quantified the expression of KIF15 at mRNA level and the expression of GAPDH was used as internal reference. The primers of KIF15 and GAPDH used for qPCR were as follows: KIF15: Forwards (5'-3') :

CTCTCACAGTTGAATGTCCTTG, Reverse(5'-3'): CTCCTTGTCAGCAGAATGAAG; GAPDH: Forwards(5'-3'): TGACTTCAACAGCGACACCCA, Reverse(5'-3'): CACCCTGTTGCTGTAGCCAAA. $2^{-\triangle \triangle C T}$ analysis program was used to analyze the expression of KIF15.

\section{Western blotting}

RIPA buffer (Invitrogen,89900,USA) was used to lyse the cell lines to obtain protein. The concentration of extracted protein was measured by using BCA kit (Solarbio,PC0020,Beijing). 25ug protein samples was separated by SDS-PAGE and then transferred to the PVDF membrane. Subsequently, the membrane was incubated with the KIF15 antibody (Abcam,ab272615,UK), GAPDH antibody (Abcam, ab181602,UK), Ki67 antibody (Abcam, ab16667,UK), or MMP9 antibody (Abcam, ab219372,UK) at $4{ }^{\circ} \mathrm{C}$ overnight. At last, the membrane was incubated with secondary antibodies for 1 hour and the bands was displayed by using ECL kit (Solarbio,PE0020,Beijing). ImageJ was performed for the semi-quantitative analysis of bands.

\section{MTT assays}

The cells in the logarithmic growth phase, which transfected with shCON or shKIF15, were collected and seeded on 96-well plates with 800 cells/well. After 6 days, MTT reagent (Solarbio,M1020,Beijing) was added into every well and cultured with cells for 2 hours. Then remove the MTT reagent, incubate with the 
cells for half an hour after adding DMSO (Solarbio,D8371,Beijing), and detect the absorbance by using a microplate reader at $570 \mathrm{~nm}$.

\section{Transwell assay}

The matrigel (Corning,354234,USA) and the pre-chilled serum-free 1640 medium were mixed on ice at a ratio of 1 to 8.60 ul diluted matrigel was added to the upper chamber (Corning,3496,USA) of transwell and 1640 medium containing $20 \%$ serum was added below the chamber. Add 9,000 cells to each chamber in a 24-well plate and incubate in the incubator for 30 hours. Discard the supernatant and stain the cells with crystal violet. Remove the remaining cells in the upper chamber at the same time. Take a photo under the microscope and Perform a cell count.

\section{Immunohistochemistry}

The tissue-embedded paraffin block was cut into sections with a thickness of $4 \mathrm{um}$. The sections were subjected to dewaxing, hydration, and antigen retrieval in order. A peroxidase blocker was added to the sections to block the effect of peroxidase. After washing with PBS, the sections were incubated with the primary antibody (KIF15, Abcam,ab272615,1:50,UK) at $4^{\circ} \mathrm{C}$ overnight. Sections were then incubated with secondary antibodies for 1 hour at $37^{\circ} \mathrm{C}$. DAB kit (Solarbio,DA1010,Beijing)was used for coloration and then hematoxylin staining was added. Finally, the slices were dehydrated and transparently treated, and neutral gum was used for sealing. Observe and take pictures under the microscope.

\section{Bioinformatics analysis}

GEPIA(http://gepia.cancer-pku.cn/) was used to analyze the PCa patients' data from TCGA(The Cancer Genome Atlas) dataset. Using the median value of KIF15 mRNA expression level in PCa patients as the cutoff value, the patients were divided into a KIF15 high expression group and a KIF15 low expression group. Kaplan-Meier method was performed to analyze the overall survival and disease-free survival in the two groups of patients. $P<0.05$ was considered statistically significant.

\section{Statistical analysis}

Statistical analysis was performed by using SPSS 22.0 software. The correlation between KIF15 expression and the clinical feature of PCa patient was analyzed by using Chi-square test. Paired t test and rank sum test were used to analyze the differences between two groups. $P<0.05$ is considered statistically significant. * indicates $\mathrm{P}<0.05$, ** indicates $\mathrm{P}<0.01$, *** indicates $\mathrm{P}<0.001$, and n.s. indicates no significant difference.

\section{Results}

\section{KIF15 was highly expressed in PCa}


In order to evaluate the KIF15 expression in PCa, at first, we collected tissues from 7 PCa patients undergoing radical prostatectomy, and tested the expression level of KIF15 in PCa tissues and prostatic tissues by using qPCR assay. As shown in Fig. 1A, KIF15 expression at mRNA level in PCa tissues is higher when compared with prostatic tissues, and it has statistical significance. Then we detected the expression at protein level of KIF15 in 3 PCa cell lines (C4-2, Lncap, and PC3) and 1 prostatic hyperplasia cell line (BPH-1) by using western blot, and found the expression of KIF15 at protein level in PCa cell lines was higher when compared with prostate hyperplasia cell line (Fig. 1B). Except that, the IHC staining was conducted to explore the KIF15 expression in 84 paraffinized prostate cancer tissues and 15 normal prostate tissues. As shown in Fig. 1C, KIF15 is mainly located in the cell cytoplasm. Compared with normal tissues, KIF15 expression levels in prostate cancer tissues are significantly increased $(P<0.001)$. In summary, KIF15 is abnormally highly expressed in PCa cells at transcription and translation levels.

\section{Suppress KIF15 expression inhibits the proliferation and invasion of PCa cells}

In order to further explore the biological effect of KIF15 in the carcinogenesis of PCa, we successfully established 2 PCa cell lines (C4-2 and Lncap) with stable knock-down of KIF15 by using shRNA. As shown in Fig. 2, verification by QPCR and WB assay found that the KIF15 expression of two PCa cell lines were successfully knocked down in both mRNA level $(P<0.01)$ and protein level $(P<0.01)$, and the knockdown efficiency is greater than $70 \%$. Previous studies have demonstrated that KIF15 was involved in the proliferation, cell apoptosis and served as novel therapeutic targets in various cancers such as osteosarcoma, melanoma and lung adenocarcinoma[17, 20-22]. Hence, we used two successfully constructed stable knockdown KIF15 cell lines to verify whether KIF15 affect the proliferation and invasion ability of prostate cancer. As shown in Fig. 3A, the MTT assay demonstrated that the proliferative capacity of PCa cells decreased significantly in two shKIF15 cell lines $(P<0.001)$. At the same time, we found that the proliferation-related marker Ki67 expression was significantly reduced in two shKIF15 cell lines by using WB assay when compared with shCON group ( $P<0.001$, Fig. 3D and 3E). The results of transwell invasion assay informed that the number of PCa cells migrating to the lower transwell chamber through matrigel decreased significantly after inhibiting KIF15 expression $(P<0.001$, Fig. 3B and Fig. 3C).Meanwhile, the invasion-related marker MMP-9 expression was also significantly

reduced after knocking down KIF15 by using western blot assay ( $P<0.001$,Fig. $3 F$ and $3 G)$. The results of in vitro experiments showed that knocking out KIF15 can effectively inhibit the proliferation and invasion ability of PCa cells.

\section{High expression of KIF15 at protein level was related with progression of Prostate cancer}

According to results of immunohistochemistry assay, the 84 PCa patients from our institution was divided into high- and low- expression KIF15 groups. Chi-square test was conducted to explore the correlation between the clinicopathological characters of PCa patients and the KIF15 expression (Table 1). Interestingly, the high KIF15 expression was related clinical T stage ( $P=0.004)$, seminal vesicle invasion $(P=0.02)$ and lymph node metastasis of $\mathrm{PCa}(P=0.03)$. However, the correlation between the 
KIF15 expresion and the age $(P=0.54)$, perioperative PSA level $(P=0.87)$ and Gleason score $(P=0.08)$ of PCa patients was not statistically significant.

\section{Overexpression of KIF15 was related with adverse prognosis of PCa patients}

Kaplan-Meier method was conducted to analyze the value of KIF15 in predicting the PCa patients prognosis. The overall survival (OS) and disease-free survival (DFS) was analyzed in 498 PCa patients from the cancer genome atlas (TCGA) database. The median value of KIF15 expression was used as the cutoff value to divide PCa patients into high KIF15 expression group and low KIF15 expression group. As shown in Fig. 4B, the DFS of high KIF15 expression was poorer than the low KIF15 expression group significantly $(P=0.0076)$. However, the OS didn't have significant difference between the two groups $(P=$ 0.17 , Fig. 4A). The results demonstrated that KIF15 expression may be a novel prognostic factor for the DFS in PCa patients which was closely related with the clinical outcomes of PCa patients.

\section{Discussion}

$\mathrm{PCa}$ is the most familiar malignant tumor with high morbidity and mortality among men in developed countries[23, 24]. With the popularization of PSA screening in China, the number of patients with prostate cancer has increased year by year, causing a huge economic and health burden to the society[25]. Due to prostate cancer is a heterogeneous multifocal solid tumor, it is difficult to accurately predict the prognosis of each PCa patient. At the same time, the tumorigenesis and occurrence of PCa has not been fully understood. Early diagnose and accurate classification of prostate cancer is of vital for urologists to develop therapeutic schedule. Therefore, it is urgent need to identify prognostic factors and potential therapeutic targets for PCa patients.

Previous studies has showed that the KIF15 expression was up-regulated in various types of malignant tumors such as osteosarcoma[17], melanoma[21], lung adenocarcinoma[22], pancreatic cancer[18] and so on. Song et al identified thirteen kinesin superfamily associated genes included KIF15 were enriched in the stage of DNA replication and cell cycle in breast cancer by using bioinformatic analysis. The overexpression of KIF15 was also associated with P53 signal pathway and mismatch repair[26]. Yu's study also confirmed that KIF15 was related with tumorigenicity of melanoma. They demonstrated that KIF15 could promote tumor growth and inhibit apoptosis of tumor cells. And the bioinformatic data found three potential targets of KIF15 included BIRC59, CDK4, and WNT5A[21] .

To our knowledge, this is first time to explore the biological effect of KIF15 in the carcinogenesis of PCa. In present research, we found that KIF15 was overexpressed in PCa when compared with normal prostatic tissues. Interestingly, the the expression of KIF15 at protein level was related with the progression of PCa such as higher clinical T stage, seminal vesicle invasion and lymph node metastasis. And the bioinformatic analysis confimred that the KIF15 high expression group in patients with PCa have an shorter DFS. Except that, the results of in vitro experiments confirmed that KIF15 can promote the proliferation and invasion ability of prostate cancer cells. This result further confirmed that KIF15 played a vital role in promoting the carcinogenesis of $\mathrm{PCa}$ at the molecular level. In a conclusion, this preliminary 
mechanism study confirmed that KIF15 can served as a novel therapeutic target for patients in PCa. Recently study showed that they developed four drug candidates targeting KIF15 by using dihydropyrrole and dihydropyrazole derivant[20]. Therefore, we believe that KIF15 inhibitors will have a promising prospect in the precise treatment of PCa patients in the near future.

\section{Conclusion}

In this study, we found that KIF15 was overexpressed in PCa. Except that, high KIF15 expression was correlated with clinical stage, seminal vesicle invasion, lymph node metastasis, and adverse DFS in PCa patients. Finally, we confirmed that KIF15 promoted proliferation and invasion of PCa cells. This study also has some limitations. The underlying mechanism of how KIF15 affects the proliferation and invasion of PCa cells should be elucidated in detail. Due to the short follow-up time and small samples of patients in our institution, no survival analysis was performed on these PCa patients. Large-scale randomized clinical controlled studies was needed to further confirm the relationship between KIF15 expression and PCa patient prognosis.

\section{Declarations}

\section{Acknowledgements:}

None

\section{Authors' contributions:}

Lin Li and Jing Hao designed and performed the research; Jin-Xiu Liu, Peng-Fei Wang, and Chao-Fei Zhao analyzed the data; Lin Li wrote the manuscript; Sheng-Yong Cai read and approved the final manuscript.

\section{Funding:}

This study was funded by Medical Science Research Projects in Hebei Province(20201519).

\section{Availability of data and materials:}

The datasets used and/or analyzed during the current study are available from the corresponding author on reasonable request.

\section{Ethics approval and consent to participate:}

Written informed consents were obtained from all participants, and this study was permitted by the Medical Ethics Committee of Tangshan Gongren Hospital.

\section{Consent for publication:}


Not applicable.

\section{Competing interests:}

The authors declare that they have no conflict of interest.

Declaration of Conflict of Interest: No.

The ethics of statement: The research was approved by the Medical Ethics Committee of Tangshan Gongren Hospital. The clinical trial registry number is GRYY-LL-2019-40.

\section{References}

1. Siegel RL, Miller KD, Jemal A. Cancer statistics, 2019. CA Cancer J Clin. 2019;69:7-34.

2. Huang $H$, Muscatelli $S$, Naslund $M$, et al. Evaluation of cancer specific mortality with surgery versus radiation as primary therapy for localized high grade prostate cancer in men younger than 60 years. The Journal of urology. 2019;201:120-8.

3. Bray F, Ferlay J, Soerjomataram I, et al Global cancer statistics 2018: GLOBOCAN estimates of incidence and mortality worldwide for 36 cancers in 185 countries. CA Cancer J Clin 2018.

4. Mariotto AB, Robin Yabroff K, Shao Y, et al. Projections of the cost of cancer care in the United States: 2010-2020. J Natl Cancer Inst. 2011;103:117-28.

5. Kimura T, Egawa S. Epidemiology of prostate cancer in Asian countries. Int J Urol. 2018;25:524-31.

6. Kirby M, Hirst C, Crawford E. Characterising the castration-resistant prostate cancer population: a systematic review. Int J Clin Pract. 2011;65:1180-92.

7. Nikhil K, Raza A, Haymour HS, et al. Aurora Kinase A-YBX1 Synergy Fuels Aggressive Oncogenic Phenotypes and Chemoresistance in Castration-Resistant Prostate Cancer. Cancers. 2020;12:660.

8. Luebke A, Ricken W, Kluth M, et al Loss of the adhesion molecule CEACAM1 is associated with early biochemical recurrence in TMPRSS2: ERG fusion positive prostate cancers. International journal of cancer 2020.

9. Wu B, Lu X, Shen H, et al Intratumoral heterogeneity and genetic characteristics of prostate cancer. International journal of cancer 2020.

10. Assadi M, Jokar N, Ghasemi M, et al. Precision Medicine Approach in Prostate Cancer. Current pharmaceutical design 2020.

11. Miki H, Okada Y, Hirokawa N. Analysis of the kinesin superfamily: insights into structure and function. Trends Cell Biol. 2005;15:467-76.

12. Hirokawa N. Organelle transport along microtubules-the role of KIFs. Trends in cell biology. 1996;6:135-41.

13. Hirokawa N, Noda Y, Okada Y. Kinesin and dynein superfamily proteins in organelle transport and cell division. Curr Opin Cell Biol. 1998;10:60-73. 
14. Khongkow P, Gomes A, Gong C, et al. Paclitaxel targets FOXM1 to regulate KIF20A in mitotic catastrophe and breast cancer paclitaxel resistance. Oncogene. 2016;35:990-1002.

15. Kato T, Wada H, Patel P, et al. Overexpression of KIF23 predicts clinical outcome in primary lung cancer patients. Lung cancer. 2016;92:53-61.

16. Xiong M, Zhuang K, Luo Y, et al. KIF20A promotes cellular malignant behavior and enhances resistance to chemotherapy in colorectal cancer through regulation of the JAK/STAT3 signaling pathway. Aging. 2019;11:11905.

17. Wu Z, Zhang H, Sun Z, et al. Knockdown of Kinesin Family 15 Inhibits Osteosarcoma through Suppressing Cell Proliferation and Promoting Cell Apoptosis. Chemotherapy. 2019;64:187-96.

18. Wang J, Guo X, Xie C, et al. KIF15 promotes pancreatic cancer proliferation via the MEK-ERK signalling pathway. Br J Cancer. 2017;117:245-55.

19. Milic B, Chakraborty A, Han K, et al. KIF15 nanomechanics and kinesin inhibitors, with implications for cancer chemotherapeutics. Proc Natl Acad Sci U S A. 2018;115:E4613-22.

20. Sebastian J. Dihydropyrazole and dihydropyrrole structures based design of Kif15 inhibitors as novel therapeutic agents for cancer. Comput Biol Chem. 2017;68:164-74.

21. Yu X, He X, Heindl LM, et al. KIF15 plays a role in promoting the tumorigenicity of melanoma. Exp Eye Res. 2019;185:107598.

22. Qiao Y, Chen J, Ma C, et al. Increased KIF15 Expression Predicts a Poor Prognosis in Patients with Lung Adenocarcinoma. Cell Physiol Biochem. 2018;51:1-10.

23. Welch HG, Albertsen PC. Reconsidering Prostate Cancer Mortality - The Future of PSA Screening. N Engl J Med. 2020;382:1557-63.

24. Zhai Z, Zheng Y, Li N, et al. Incidence and disease burden of prostate cancer from 1990 to 2017: Results from the Global Burden of Disease Study 2017. Cancer. 2020;126:1969-78.

25. Liu J, Dong B, Qu W, et al. Using clinical parameters to predict prostate cancer and reduce the unnecessary biopsy among patients with PSA in the gray zone. Sci Rep. 2020;10:5157.

26. Song X, Zhang T, Wang X, et al. Distinct Diagnostic and Prognostic Values of Kinesin Family Member Genes Expression in Patients with Breast Cancer. Med Sci Monit. 2018;24:9442-64.

\section{Figures}




\section{Figure 1}

A

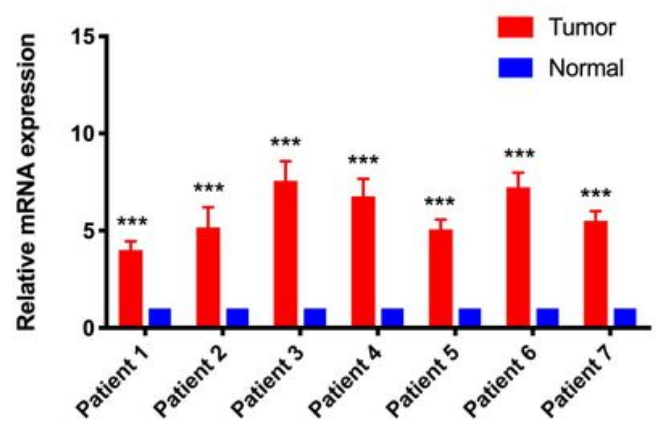

C

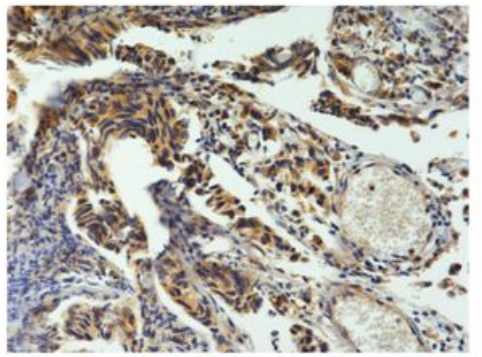

Tumor (High)

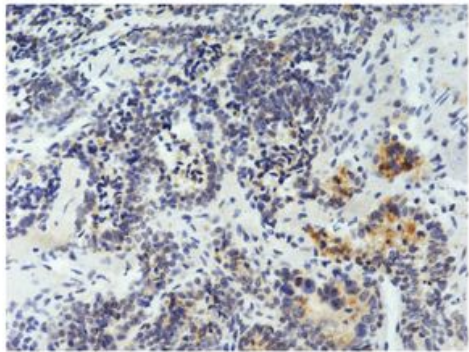

Tumor (Low)

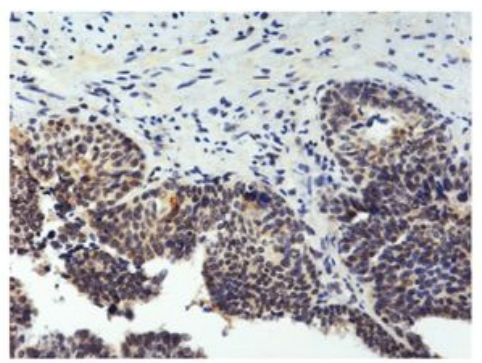

Tumor (Medium)

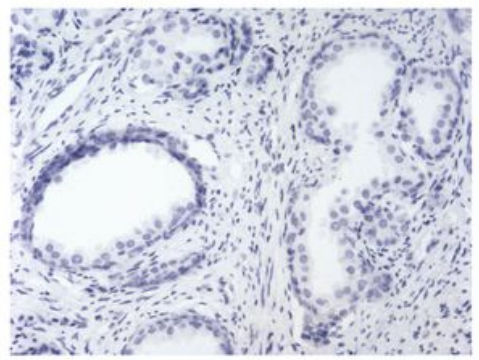

Normal tissues
B

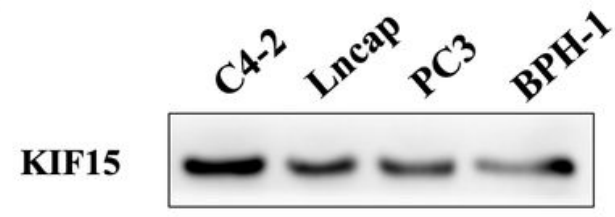

GAPDH
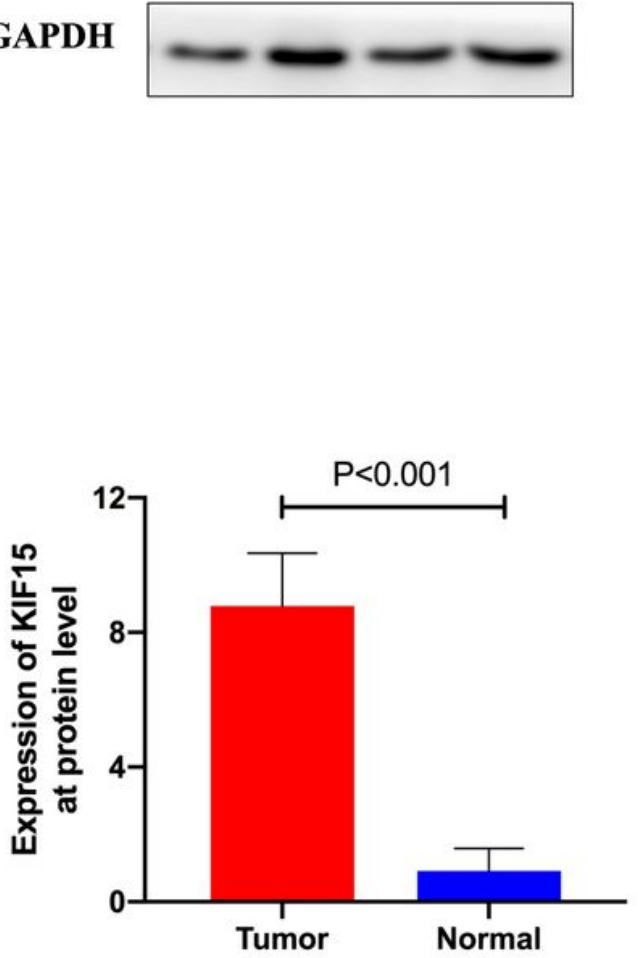

\section{Figure 1}

KIF15 expression was upregulated in prostate cancer compared with normal tissues A) The expression of KIF15 at mRNA level in PCa patients by qPCR. B) Expression of KIF15 in 3 PCa cell lines and 1 Prostate hyperplastic epithelium cells line by using western blot. C) Representative IHC staining images of KIF15 and the associated statistics in PCa tissues and normal prostate tissues $(P<0.001)$. 
Figure 2

A
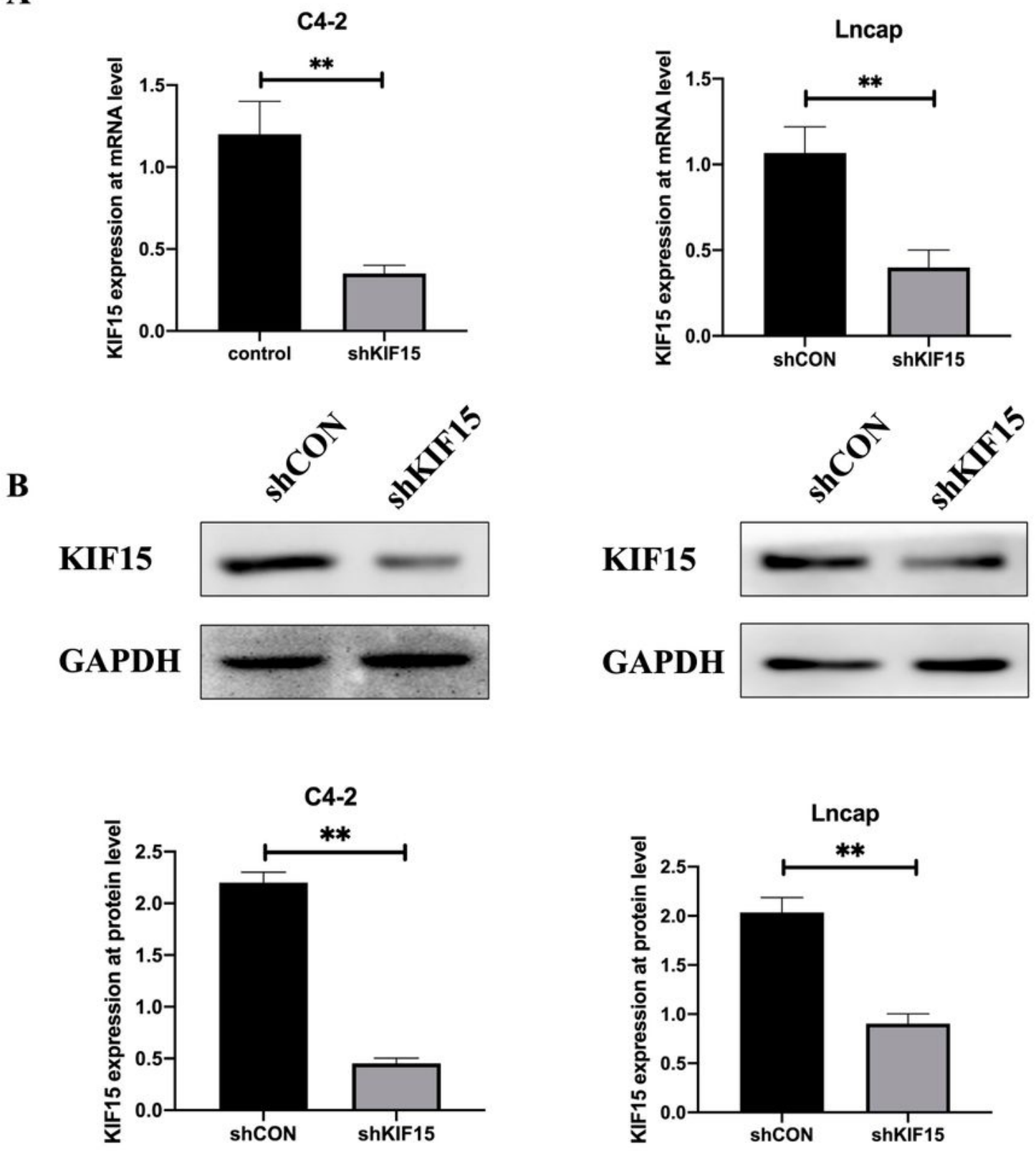

Figure 2

KIF15 was successfully knockdown in both C4-2 and Lncap cell lines by shRNA A) The shKIF15 lentiviralbased vector was transfected into A-549 and PC-9 cell lines. The expression of KIF15 at mRNA level was verified by $q P C R$. The histogram is a semi-quantitative analysis of $q P C R$ data $(* * P<0.01)$. B) After knockdown KIF15 by shRNA, the expression of KIF15 at protein level was verified by western blot. The histogram is a semi-quantitative analysis of western blot data $(* * P<0.01)$. 
Figure 3
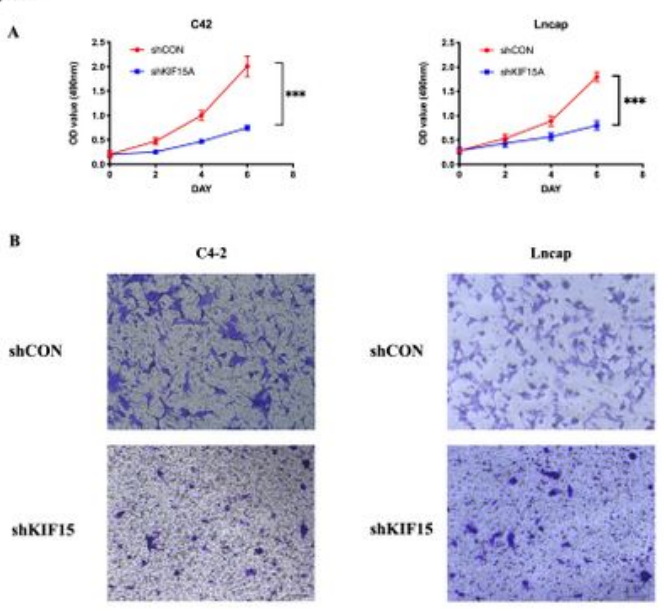

c
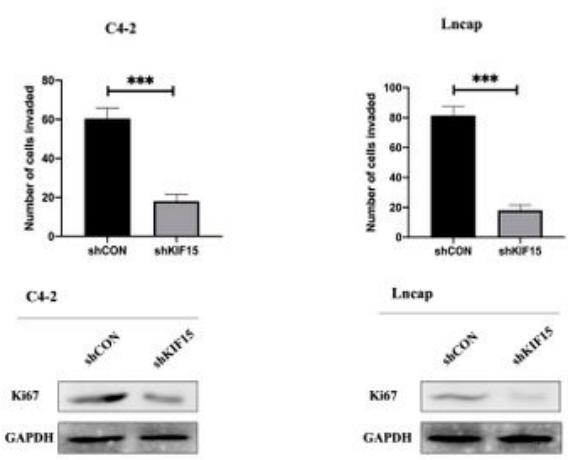

Lncap

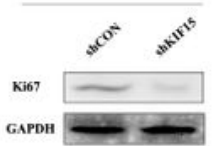

E

C4-2

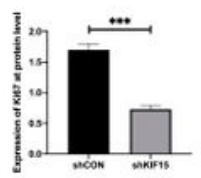

Lncap
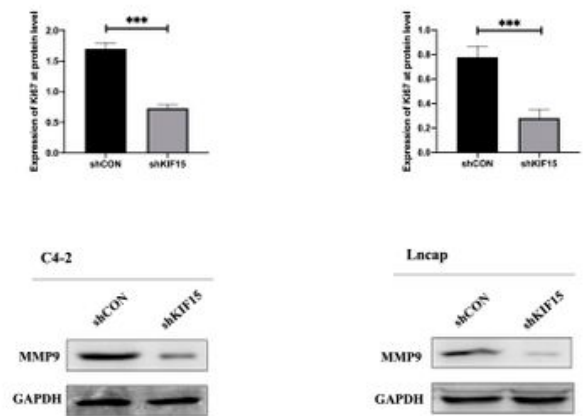

G
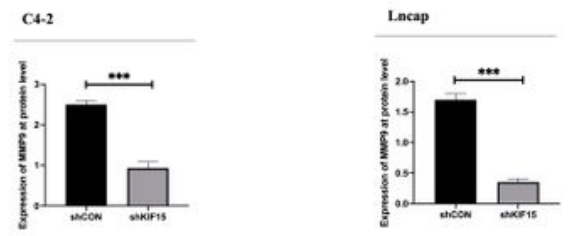

\section{Figure 3}

Knockdown of KIF15 inhibits the proliferation and invasion abilities of PCa cells. A) MTT assay was used to estimate the growth in both C4-2 and Lncap cell lines after knocking down KIF15. The absorbance value was measured at $490 \mathrm{~nm}(* \star * P<0.001)$. B) Transwell assay was used to estimate the invasion ability of C4-2 and Lncap cell lines after knocking down KIF15. C) Histogram was used to analyze the number of invasion cells ( $* * * P<0.001)$. D) The expression levels of Ki67 was detected by western blot 
after knocking down KIF15 in both C4-2 and Lncap cell lines. E) The histogram is a semi-quantitative analysis of the Ki67 expression at protein level( $(* \star \mathrm{P}<0.001)$. F) The expression levels of MMP9 was detected by western blot after knocking down KIF15 in both C4-2 and Lncap cell lines. E) The histogram is a semi-quantitative analysis of the MMP9 expression at protein level $(* \star * P<0.001)$.

\section{Figure 4}
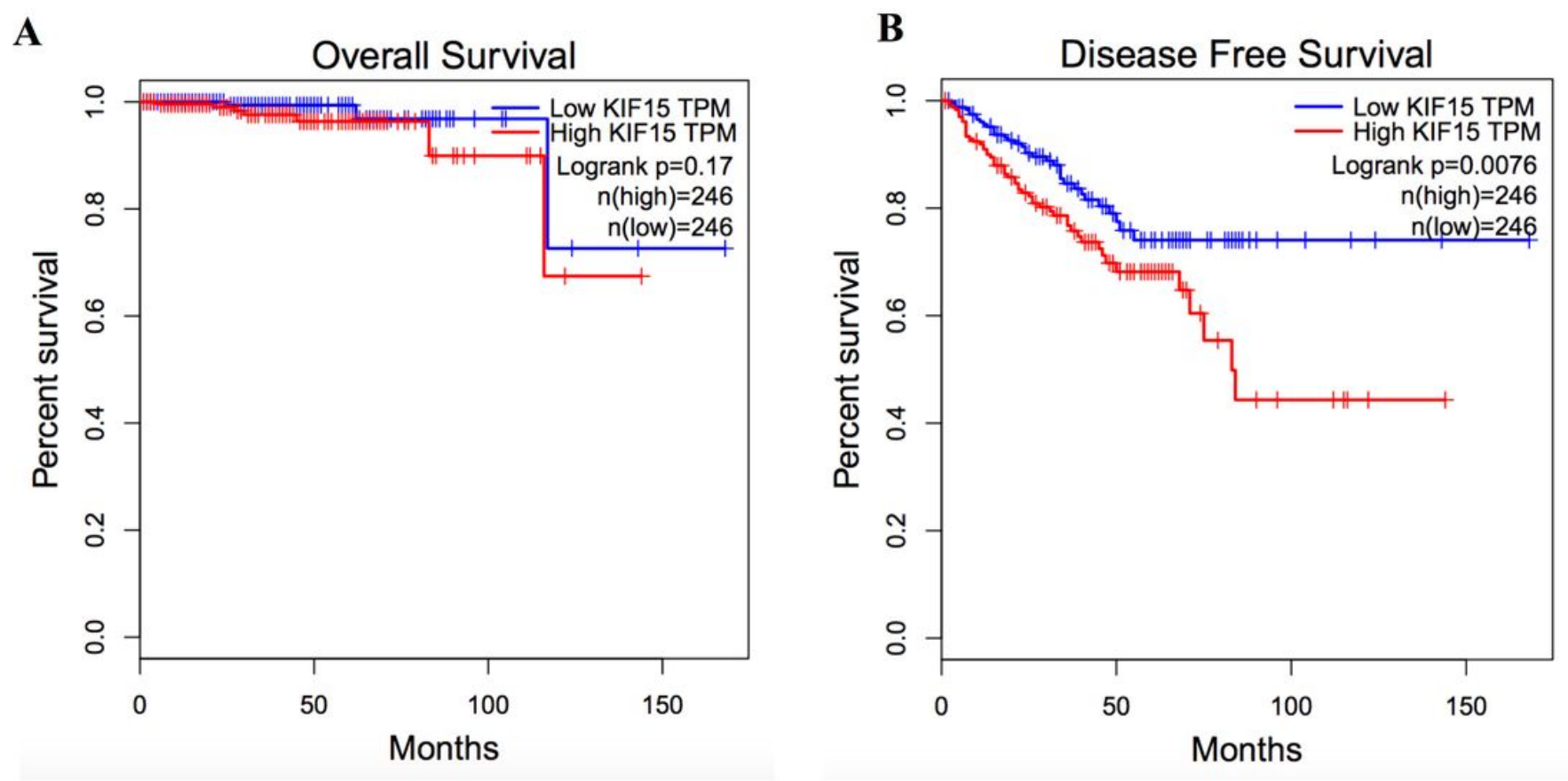

\section{Figure 4}

Overexpression of KIF15 indicates an poor prognosis in PCa patients. A) Overall survival analysis of $\mathrm{PCa}$ patients from TCGA dataset by Kaplan-Meier method $(P=0.17)$. B) Disease-free survival analysis of $P C a$ patients TCGA dataset by Kaplan-Meier method $(P=0.0076)$.

\section{Supplementary Files}

This is a list of supplementary files associated with this preprint. Click to download.

- Table1.xlsx 